\title{
INTERSECTION THEOREMS FOR POSITIVE SETS ${ }^{1}$
}

\author{
WOLFHARD HANSEN AND VICTOR KLEE
}

Introduction. Helly's intersection theorem [7] asserts that if $\mathbf{C}$ is a finite family of convex sets in $R^{d}$ with $\cap \mathbf{C}=\varnothing$ then $\boldsymbol{C}$ admits a subfamily $K$ with $\cap K=\varnothing$ and $|K| \leqq d+1$. The shortest proof, due to Radon [9], is based on the fact that a subset of $R^{d}$ is affinely independent if and only if it does not contain disjoint sets whose convex hulls intersect. Here a similar approach leads to short proofs of old and new intersection theorems for positive sets.

Throughout this note, $E$ denotes a vector space over an ordered field. When $E$ is said to be $d$-dimensional it should be understood that $d$ is finite. A subset $P$ of $E$ is called positive provided that $\alpha x+\beta y \in P$ whenever $x, y \in P$ and $\alpha, \beta \geqq 0$; equivalently, $P$ is a convex cone with apex 0 . (When $E=R^{d}$, the intersections of positive sets with the unit sphere are precisely the sets which are spherically convex in one of the common meanings of that term. Thus for real vector spaces our theorems could be stated alternatively in terms of spherically convex sets.) The positive hull pos $X$ of a set $X \subset E$ is the intersection of all positive sets containing $X$; equivalently, it is the set of all points of the form $\sum_{x \in X} \lambda_{x} x$ with $\lambda_{x} \geqq 0$ for all $x$ and $\lambda_{x}=0$ for all but finitely many $x$. Note that $\operatorname{lin} X=\operatorname{pos} X-\operatorname{pos} X$, where lin $X$ is the linear hull of $X$.

Strong positive independence. A subset $X$ of $E \sim\{0\}$ is called strongly positively independent provided that pos $Y \cap$ pos $Z \subset\{0\}$ whenever $Y$ and $Z$ are disjoint subsets of $X$. This notion was introduced by McKinney [8] and characterized in various ways by him, Bonnice and Klee [1], and Reay [10]. The most useful characterization is the following, proved by McKinney when pos $X=\operatorname{lin} X$. Our proof is considerably shorter than his.

Theorem (McKinney). A subset $X$ of $E$ is strongly positively independent if and only if $E$ can be expressed as a direct sum of linear subspaces, $E=E_{0} \oplus \oplus_{\alpha \in A} E_{\alpha}$, in such a way that

(a) $X \subset E_{0} \cup \cup_{\alpha \in A} E_{\alpha}$,

(b) $X \cap E_{0}$ is linearly independent,

(c) for each $\alpha \in A$ the subspace $E_{\alpha}$ is finite-dimensional and $X \cap E_{\alpha}$

Received by the editors December 11, 1968.

1 This paper was written at the University of Washington when the first author was there as a visiting faculty member. Work of the second author was supported in part by the Boeing Scientific Research Laboratories and in part by the Office of Naval Research. 
consists of the points of a linear basis for $E_{\alpha}$ together with a sum of negative multiples of these points.

Proof. For the "if" part it suffices to note that each of the intersections $X \cap E_{\alpha}$ is strongly positively independent. For the "only if" part, consider a strongly positively independent subset $X$ of $E$ and let $B$ be a linear basis for $X$-that is, $B$ is linearly independent and $B \subset X \subset \operatorname{lin} B$. Let $A=X \sim B$. For each point $x$ of $A$ there is a unique scalar function $\lambda^{x}$ on $B$ such that $\lambda_{b}^{x}=0$ for all but finitely many $b \in B$ and $x=-\sum_{b \in B} \lambda_{b}^{x} b$. Let $B_{x}=\left\{b \in B: \lambda_{b}^{x}>0\right\}$. Then

$$
x+\sum_{b \in B_{x}} \lambda_{b}^{x} b=\sum_{b \in B \sim B_{x}}\left(-\lambda_{b}^{x}\right) b
$$

and it follows from strong positive independence that both sides of (1) are equal to 0 . Note that $B_{x} \cap B_{y}=\varnothing$ whenever $x, y \in A$ with $x \neq y$. For suppose the contrary and let $\mu=\max \left\{\lambda_{b}^{x} / \lambda_{b}^{y}: b \in B_{y}\right\}>0$. Then

$$
x+\sum_{b \in B_{x} \sim B_{y}} \lambda_{b}^{x} b=\mu y+\sum_{b \in B_{x} \cap B_{y}}\left(\mu \lambda_{b}^{y}-\lambda_{b}^{x}\right) b+\sum_{b \in B_{y} \sim B_{x}} \lambda_{b}^{y} b
$$

and it follows from strong postive independence that both sides are 0 . Referring to (1), we conclude that $\sum_{b \in B_{x} \cap B_{y}} \lambda_{b}^{x} b=0$, a contradiction implying $B_{x} \cap B_{y}=\varnothing$.

Now let $B^{\prime}$ be a linear basis for $E$ containing $B$, let

$$
E_{0}=\operatorname{lin}\left(B^{\prime} \sim \bigcup_{x \in A} B_{x}\right)
$$

and for each $x \in A$ let $E_{x}=\operatorname{lin} B_{x}$. Then $E=E_{0} \oplus^{\oplus} \oplus_{x \in A} E_{x}$ and conditions (a), (b) and (c) are satisfied.

The subspaces $E_{\alpha}$ in the above decomposition are uniquely determined by $X$, for they are exactly those finite-dimensional subspaces $L$ of $E$ such that $L=\operatorname{pos}(X \cap L)$ and $1+\operatorname{dim} L=|X \cap L|$. (By Davis [3], McKinney [8] and others they have been called the minimal subspaces associated with $X$.) The set $X \cap E_{0}$ is also determined by $X$, as is $E_{0}$ itself when lin $X=E$. When $E$ is finite-dimensional the cardinalities $\left|X \cap E_{0}\right|$ and $\left|X \cap E_{\alpha}\right|$ can be arranged in a finite sequence which starts with $\left|X \cap E_{0}\right|$ and thereafter lists the numbers $\left|X \cap E_{\alpha}\right|$ in increasing order and with proper multiplicity. This sequence will be called the invariant of $X$ in $E$. For example, the sequence $(1 ; 2,2,3)$ is the invariant in $R^{5}$ of the eight-pointed strongly positively independent set represented by the columns of the follow- 
ing matrix:

$\begin{array}{rrrrrrrr}1 & 0 & 0 & 0 & 0 & 0 & 0 & 0 \\ 0 & 1 & -1 & 0 & 0 & 0 & 0 & 0 \\ 0 & 0 & 0 & 1 & -1 & 0 & 0 & 0 \\ 0 & 0 & 0 & 0 & 0 & 1 & 0 & -1 \\ 0 & 0 & 0 & 0 & 0 & 0 & 1 & -1\end{array}$

The term invariant is justified by the first part of the following theorem, whose straightforward proof is left to the reader.

Theorem. Suppose that $E$ is $d$-dimensional. Two strongly positively independent subsets $X$ and $Y$ of $E$ have the same invariant in $E$ if and only if $E$ admits a linear automorphism carrying the rays from the origin through the points of $X$ onto the rays from the origin through the points of $Y$. A sequence $\left(t_{0} ; t_{1}, \cdots, t_{r}\right)$ of integers is the invariant in $E$ of some strongly positively independent set of cardinality $n$ if and only if the following conditions are all satisfied: $0 \leqq t_{0} ; 2 \leqq t_{1} \leqq \cdots \leqq t_{r}$; $n=\sum_{0}^{r} t_{i} \leqq d+r$.

With slight modifications the above theorem can be extended to the infinite-dimensional case. From the theorem's second assertion it follows readily that $d+[d / j]$ is the maximum cardinality of strongly positively independent subsets of $E$ in which each $j$-pointed set is linearly independent.

A subset $C$ of $E$ is called a cross basis for $E$ (called a maximal positive basis by Davis [3] and McKinney [8]) provided that $C$ consists of the points of a linear basis for $E$ together with a negative multiple of each of these points. The following is an immediate consequence of the preceding theorems.

Corollary. Suppose that $E$ is d-dimensional and $X \subset E \sim\{0\}$. If $|X|>2 d$ then $X$ contains two disjoint subsets whose positive hulls have a common nonzero point. The same is true when $|X|=2 d$ unless $X$ is a cross basis for $E$.

Intersection theorems. When $X \subset E \sim\{0\}$ we will say that the sets of the form $\operatorname{pos}(X \sim\{x\})$, for $x \in X$, are associated with $X$. Note that all the sets associated with a cross basis are closed halfspaces. Before proving the main intersection theorems, we illustrate the method to be employed by proving the following result of Robinson [11].

Corollary (Robinson). Suppose that $E$ is d-dimensional and $P$ is 
a finite family of positive sets in $E$ with $\cap P \subset\{0\}$. Then $P$ admits a subfamily $Q$ with $\cap Q \subset\{0\}$ and $|Q| \leqq 2 d$. Indeed, there is such $a \mathbf{Q}$ with $|\boldsymbol{Q}|<2 d$ unless $\boldsymbol{P}$ consists of the $2 d$ halfspaces associated with $a$ cross basis for $E$ or of such halfspaces together with $E$ itself.

Proof. Let $Q_{1}, \cdots, Q_{n}$ be distinct members of $P$ forming a subfamily $Q$ with $\cap Q \subset\{0\}$ and $|Q|$ a minimum. For each $i$ there is a nonzero point $x_{i} \in \bigcap_{j \neq i} Q_{i}$. If $x_{i}=x_{k}$ with $i \neq k$ then $x_{i} \in \cap Q$. We may assume, therefore, that the $x_{i}$ 's are all distinct and let $X$ denote the $n$-pointed set $\left\{x_{1}, \cdots, x_{n}\right\}$. If $X$ contains two disjoint sets whose positive hulls have a common nonzero point $v$ then $v \in \cap Q$, an impossibility since $\cap Q \subset\{0\}$. From the preceding corollary it follows that $n<2 d$ or $n=2 d$ and $X$ is a cross basis. It remains to examine the nature of $P$ in the latter instance. Plainly $Q_{i}$ is a halfspace associated with the cross basis $X$, for $Q_{i}$ is positive and $X \sim\left\{x_{i}\right\} \subset Q_{i} \neq E$. Consider an arbitrary member $P$ of $P \sim Q$. If $P \supset X$ then of course $P=E$. If there is an $i$ for which $x_{i} \notin P$, then $\{P\} \cup\left(Q \sim\left\{Q_{i}\right\}\right)$ is a subfamily of $P$ with intersection $C\{0\}$ and by the earlier reasoning is the set of all halfspaces associated with a cross basis for $E$. It then follows that $P=Q_{i}$.

The special case of the above result in which $P$ consists of closed halfspaces has (or its polar equivalent has) been proved by Steinitz [12], Dines and McCoy [4], Robinson [11], Gustin [6], Gale [5] and others, and has been applied to systems of linear inequalities by Blumenthal [0]. The polar equivalent asserts that if pos $X=E$ then pos $Y=E$ for some $Y \subset X$ with $|Y| \leqq 2 d$; further, there is such a $Y$ with $|Y|<2 d$ unless $X$ is a cross basis for $E$. See Danzer, Grünbaum, and Klee [2] for references to related results.

The statements of our main theorems will require some more definitions. For any set $Z$ let $d_{L}(Z)$ denote the maximum of the dimensions of the linear subspaces contained in $Z$. For any family $\boldsymbol{Z}$ of sets let

$$
k_{*}(\boldsymbol{Z})=\min _{\boldsymbol{Z} \in \boldsymbol{Z}} d_{L}(Z), \quad k^{*}(\boldsymbol{Z})=\max _{\boldsymbol{Z} \in \boldsymbol{Z}} d_{L}(Z),
$$

and

$$
l(\boldsymbol{Z})=d_{L}(\mathrm{U} \boldsymbol{Z})
$$

The family $P$ is said to be compatible with the invariant $\left(t_{0} ; t_{1}, \cdots\right.$, $t_{r}$ ) provided that there exists a strongly positively independent set $X$ in $E$ with this invariant such that each member of $\boldsymbol{P}$ contains a member of $A_{X}$, the family of all sets associated with $X$.

Theorem. Suppose that $E$ is a d-dimensional space, $0 \leqq k \leqq l \leqq d$, and 
$m=\min (k+1, l)$. Let $Q$ be a finite family of positive sets in $E$ that is minimal with respect to having $\cap Q \subset\{0\}$. If $k_{*}(Q) \leqq k$ and $l(Q) \leqq l$ then $|\boldsymbol{Q}| \leqq d+m$, with equality if and only if $\boldsymbol{Q}$ is compatible with

$$
\begin{gathered}
(d-m-s ; \quad 2, \cdots, 2,2+s) \\
(m-12 ' s)
\end{gathered}
$$

for some $0 \leqq s \leqq l-m$.

Proof. Let $Q_{1}, \cdots, Q_{n}$ be the $n$ members of $Q$ and let $X=\left\{x_{1}, \cdots, x_{n}\right\}$, where for each $i$ the point $x_{i}$ is such that $0 \neq x_{i} \in \bigcap_{j \neq i} Q_{i}$. Let $\left(t_{0} ; t_{1}, \cdots, t_{r}\right)$ be the invariant of the strongly positively independent set $X$ and let

$$
E=E_{0} \oplus E_{1} \oplus \cdots \oplus E_{r}
$$

be the direct sum decomposition of $E$ described in the first theorem. Since $X \sim\left\{x_{i}\right\} \subset Q_{i}$ for all $i$, it follows that

$$
r-1 \leqq \sum_{1}^{r-1}\left(t_{i}-1\right)=k_{*}\left(A_{X}\right) \leqq k_{*}(\boldsymbol{Q}) \leqq k
$$

And since, for $j>0$, each point of $E_{j}$ is a positive combination of a proper subset of $X \cap E_{j}$, it follows that

$$
E_{1} \oplus \cdots \oplus E_{r} \subset \cup_{A_{X}} \subset \cup_{Q}
$$

and

$$
r \leqq \sum_{1}^{r}\left(t_{i}-1\right)=l\left(A_{X}\right) \leqq l(Q) \leqq l .
$$

We conclude, therefore, that $r \leqq \min (k+1, l)$ and $n \leqq d+r \leqq d+m$. Note that the inequality $n \leqq d+m$ is all that is required for the corollary below.

Suppose now that $n=d+m$, whence $r=m$. If $t_{r-1}>2$ then $k \geqq k_{*}(Q) \geqq r=\min (k+1, l)$, whence $k=l$ and $l \geqq l(Q) \geqq r+1=l+1$, a contradiction. It follows that $t_{1}=\cdots=t_{r-1}=2$. Furthermore,

$$
t_{r} \leqq 1+l-\sum_{1}^{r-1}\left(t_{i}-1\right)=1+l-(m-1)=2+l-m .
$$

Let $s=t_{r}-2$. Then $0 \leqq s \leqq l-m$ and

$$
t_{0}=n-\sum_{1}^{r} t_{i}=d+m-2(m-1)-(2+s)=d-m-s .
$$

Hence the invariant of $X$ is 


$$
\begin{gathered}
(d-m-s, \quad 2, \cdots, 2, \quad 2+s) \\
(m-12 \text { 's })
\end{gathered}
$$

or simply (d) if $m=0$. Plainly $|\boldsymbol{Q}|=d+m$ if $Q$ is compatible with such an invariant for some $0 \leqq s \leqq l-m$, and that completes the proof.

Corollary (Robinsin). Suppose that $E$ is d-dimensional and $P$ is a finite family of positive sets in $E$ with $\cap P \subset\{0\}$ and $k^{*}(P) \leqq k$. Then $\boldsymbol{P}$ admits a subfamily $\boldsymbol{Q}$ with $\cap Q \subset\{0\}$ and $|\boldsymbol{Q}| \leqq d+k+1$.

Proof. Choose $P_{0} \in P$ with $d_{L}\left(P_{0}\right) P_{0} \leqq k$ and let $S$ be a subfamily of $P \sim\left\{P_{0}\right\}$ that is minimal with respect to having $P_{0} \cap(\cap S) \subset\{0\}$. Let $\boldsymbol{M}=\left\{S \cap P_{0}: S \in S\right\}$. Then $l(\boldsymbol{M}) \leqq k$ and $\boldsymbol{M}$ is minimal with respect to having $\cap M \subset\{0\}$, so it follows from the preceding theorem that $|M| \leqq d+k$. But then $\left|\left\{P_{0}\right\} \cup S\right| \leqq d+k+1$.

THEOREM. Let the hypotheses of the preceding theorem be strengthened by requiring $k^{*}(\boldsymbol{Q}) \leqq k$. Then

(a) when $m=d$ or $k=l,|\mathbf{Q}|=d+m$ if and only if $\mathbf{Q}$ is compatible with $(d-m ; 2, \cdots, 2)(m 2$ 's);

(b) when $k=0$ and $l=d,|\boldsymbol{Q}|=d+m$ if and only if $\boldsymbol{Q}$ is compatible with $(0 ; d+1)$;

(c) when $m<d$ and $0<l-k<d,|Q| \leqq d+m-1=d+k$, with equality if and only if $\mathbf{Q}$ is compatible with $(d-k ; 2, \cdots, 2)(k 2$ 's) or $k=d-2$ and $Q$ is compatible with $(0 ; 2, \cdots, 2)(d-12$ 's).

Proof. If $|\boldsymbol{Q}|=d+m$ then $\boldsymbol{Q}$ is compatible with

$$
\begin{gathered}
(d-m-s ; 2, \cdots, 2,2+s) \\
(m-12 \text { 's })
\end{gathered}
$$

for some $0 \leqq s \leqq l-m$. There are the following cases to consider:

(i) $m+s<d$. Then $k \geqq k^{*}\left(\boldsymbol{A}_{X}\right)=\sum_{1}^{r}\left(t_{i}-1\right)=m+s$, whence $m \leqq k$, $k=l$, and $s=0$.

(ii) $m=d$ and $s=d-m=0$.

(iii) $1=m$ and $s=d-1>0$. Then $s \leqq l-m$ implies $l=d$ and $k=0$.

(iv) $1<m<d$ and $s=d-m$ is impossible, for it implies $d=l$ and $k \geqq k^{*}\left(\boldsymbol{A}_{X}\right)=m-1+s=d-1$, whence $m=d$.

That settles the "only if" parts of (a) and (b), the first part of (c), and supplies all the information needed for the corollary below. The "if" parts of (a) and (b) are obvious.

For the remainder of the proof we assume $m<d, 0<l-k<d$, and $|Q|=d+k$. Note first that $k \leqq r \leqq k+1$, where the left-hand inequality 
follows from $n \leqq d+r$ and the right-hand inequality from

$$
k \geqq k^{*}\left(A_{X}\right) \geqq \sum_{2}^{r}\left(t_{i}-1\right) \geqq r-1 .
$$

Now suppose first that $t_{0}>0$. Then

$$
k \geqq k^{*}\left(A_{X}\right)=\sum_{1}^{r}\left(t_{i}-1\right) \geqq r+t_{r}-2,
$$

whence $r=k$ and $t_{i}=2$ for all $i>0$. Hence $X$ 's invariant is $(d-k$; $2, \cdots, 2)\left(k 2\right.$ 's). Suppose next that $t_{0}=0$ and $r=k+1$. Then

$$
k \geqq k^{*}\left(A_{X}\right)=\sum_{2}^{r}\left(t_{i}-1\right) \geqq k+t_{r}-2,
$$

whence $t_{i}=2$ for all $i>0$ and $d+k=\sum_{1}^{r} t_{i}=2(k+1)$. Hence $k=d-2$ and $X$ has invariant $(0 ; 2, \cdots, 2)(d-12$ 's). Suppose, finally, that $t_{0}=0$ and $r=k$. As $t_{r-1}>2$ or $t_{r}>3$ would imply

$$
k \geqq k^{*}\left(A_{X}\right) \geqq \sum_{2}^{r}\left(t_{i}-1\right) \geqq k+1,
$$

it follows that $t_{1}=\cdots=t_{r-1}=2$ and $t_{r}=2+s$ with $0 \leqq s \leqq 1$. This implies

$$
d+k=\sum_{1}^{r} t_{i}=2(k-1)+2+s \leqq 2 k+1
$$

and hence $k \geqq d-1$, contradicting the fact that $k<l$ and $m<d$. Thus it cannot happen that $t_{0}=0$ and $r=k$, and the discussion of (c)'s "only if" part is complete. Again, the "if" part is obvious.

COROLLARY. Let the hypotheses of the preceding corollary be strengthened by requiring that $k^{*}(P) \leqq k$ and that $0<k<d-1$ or $l(P)<d$. Then $P$ admits a subfamily $\boldsymbol{Q}$ with $\cap Q \subset\{0\}$ and $|\boldsymbol{Q}| \leqq d+k$.

Proof. Let $Q$ be a subfamily of $P$ that is minimal with respect to having $\cap Q \subset\{0\}$. Apply the theorem just proved.

\section{REFERENCES}

0. L. M. Blumenthal, Metric methods in linear inequalities, Duke Math. J. 15 (1948), 955-966.

1. W. Bonnice and V. Klee, The generation of convex hulls, Math. Ann. 152 (1963), 1-29.

2. L. Danzer, B. Grünbaum, and V. Klee, Helly's theorem and its relatives, Proc. Sympos. Pure Math., Vol. 7, Amer. Math. Soc., Providence, R. I., 1963, pp. 101-180. 
3. C. Davis, Theory of positive linear dependence, Amer. J. Math. 76 (1954), 733746.

4. L. L. Dines and N. H. McCoy, On linear inequalities, Trans. Roy. Soc. Canada 27 (1933), 37-70.

5. D. Gale, Linear combinations of vectors with non-negative coefficients, Amer. Math. Monthly 59 (1952), 46-47.

6. W. Gustin, On the interior of the convex hull of a Euclidean set, Bull. Amer. Math. Soc. 53 (1947), 299-301.

7. E. Helly, Über Mengen konvexer Körper mit gemeinschaftlichen Punkten, Jber. Deutsch. Mat.-Verein. 32 (1923), 175-176.

8. R. L. McKinney, Positive bases for linear spaces, Trans. Amer. Math. Soc. 103 (1962), 131-148.

9. J. Radon, Mengen konvexer Körper, die einen gemeinsamen Punkt enthalten, Math. Ann. 83 (1921), 113-115.

10. J. R. Reay, Unique minimal representations with positive bases, Amer. Math. Monthly 73 (1966), 253-261.

11. C. V. Robinson, Spherical theorems of Helly type and congruence indices of spherical caps, Amer. J. Math. 64 (1942), 260-272.

12. E. Steinitz, Bedingt konvergente Reihen und konvexe Systeme. II, J. Reine Angew. Math. 144 (1914), 1-40.

UNIVERSITY OF ERLANGEN-NÜRNBERG AND

UNIVERSITY OF WASHINGTON 